ORIGINAL ARTICLE

\title{
Relationship between Trendelenburg tilt and internal jugular vein diameter
}

\author{
S Clenaghan, R E McLaughlin, C Martyn, S McGovern, J Bowra
}

Emerg Med J 2005;22:867-868. doi: 10.1136/emj.2004.019257

See end of article for authors' affiliations

.....................

Correspondence to: S Clenaghan, Ulster Hospital, Upper

Newtownards Road Belfast, Northern Ireland; stepclen@hotmail.com

Accepted for publication 21 January 2005

\begin{abstract}
Objectives: To evaluate the relationship between Trendelenburg tilt and internal jugular vein (IJV) diameter, and to examine any cumulative effects of tilt on the IJV diameter.

Methods: Using a tilt table, healthy volunteers were randomised to Trendelenburg tilts of $10^{\circ}, 15^{\circ}, 20^{\circ}$, $25^{\circ}$, and $30^{\circ}$. Ultrasound was used to measure and record the lateral diameter of the right IJV at the level of the cricoid cartilage. Following each reading the table was returned to the supine position. Balanced randomisation was used to assess cumulative tilt effects.

Results: A total of 20 healthy volunteers were recruited (10 men, 10 women). Mean supine IJV diameter was $13.5 \mathrm{~mm}(95 \% \mathrm{Cl} 12.8$ to 14.1$)$ and was significantly greater at $10^{\circ}(15.5 \mathrm{~mm}, 95 \% \mathrm{Cl} 14.9$ to 16.1$)$. There was no significant difference between $10^{\circ}$ and greater angles of tilt. The effect of the previous angle of tilt did not prove to be statistically significant.

Conclusion: Increasing the degree of Trendelenburg tilt increases the lateral diameter of the IJV. Even a $10^{\circ}$ tilt is effective. The cumulative effect of tilt (that is, the effect of the previous angle) is not significant. Ultrasound guided cannulation is ideal, but in its absence Trendelenburg tilt will increase IJV diameter and improve the chance of successful cannulation. While $25^{\circ}$ achieved optimum distension, this may not be practical and may be detrimental (for example, risk of raised intracranial pressure).
\end{abstract}

$\mathrm{T}$ he UK National Institute of Clinical Excellence (NICE) guidelines ${ }^{1}$ recommend the use of real time ultrasound guidance for internal jugular vein (IJV) cannulation. However, a recent study has shown that the majority of emergency departments (EDs) in the island of Ireland do not use ultrasound. ${ }^{2}$ Although strictly speaking these departments do not fall under the jurisdiction of NICE, the pattern of ultrasound usage in EDs is likely similar to that in mainland UK. In the absence of ultrasound guidance the Trendelenburg position is often used in IJV cannulation to increase the diameter of the IJV.

Our study aimed to evaluate the relationship between Trendelenburg tilt and the IJV diameter and to examine any cumulative effects of tilt on the IJV diameter.

\section{METHOD}

A statistical analysis before the study predicted that 20 subjects would be required for sufficient power, and compared favourably with other similar studies. ${ }^{3}$ The procedure was carried out by a single examiner (SC) using healthy volunteers selected at random. The subjects had no history of neck problems or previous IJV cannulation.

Each subject was placed supine on a tilt table, with an inbuilt clinometer that was raised and lowered on an electric motor. The level of the cricoid cartilage was marked and the right IJV was identified using real time ultrasound (Medison 128 BW high frequency linear probe). Lateral IJV diameter was measured at this level over three respiratory cycles and the maximum diameter was recorded prior to tilt. The subject then was tilted to $10^{\circ}, 15^{\circ}, 20^{\circ}, 25^{\circ}$, and $30^{\circ}$ Trendelenburg. For each angle, subjects were kept in position for 30 seconds prior to further measurement of maximum IJV diameter (again over three respiratory cycles) and then returned to the supine position before being placed in the next angle. The actual order of angles was randomly allocated for each subject using a balanced randomisation table, ${ }^{4}$ so that we could analyse the effects of prior angle and cumulative tilt.
We recorded and printed ultrasound images of maximal IJV diameter for each subject at each angle. The results were collated and we calculated the mean IJV diameter for each angle studied.

A factorial analysis of variance (including terms for angle, subject, previous angle, and period) performed with SPSS version 11 showed that previous angle $(p=0.76)$ and period $(p=0.68)$ were not significant. This also allowed us to include the zero tilt results that were always performed in the first period. We compared angles in pairs using NewmanKeuls multiple range tests and we also described the relationship between diameter and tilt angle using polynomial trends.

\section{RESULTS}

We recruited a total of 20 subjects ( 10 men, 10 women; age range 22-57 years). Clear ultrasound images were obtained and no anatomical anomalies were encountered.

The mean supine IJV diameter ranged from $13.5 \mathrm{~mm}$ at $0^{\circ}$ tilt to $16.7 \mathrm{~mm}$ at $25^{\circ}$ tilt (table 1 , fig 1 ). Mean IJV diameter increased significantly at $10^{\circ}$ tilt. There was a non-significant trend to increased diameter with greater angles of tilt, with a maximum at $25^{\circ}$.

\section{DISCUSSION}

In the ED, patients who require emergent central venous cannulation are those in need of rapid infusion of fluids or drugs, or monitoring of central venous pressure because of cardiovascular instability. Cannulation in such patients is often difficult (for example, due to intravascular depletion) and may have an increased risk of complications such as failed cannulation, arterial puncture, haematoma, and pneumothorax.

In this context, institutions such as NICE recommend ultrasound guidance for central vein cannulation in order to

Abbreviations: $E D$, emergency department; IJV, internal jugular vein; NICE, National Institute for Clinical Excellence 


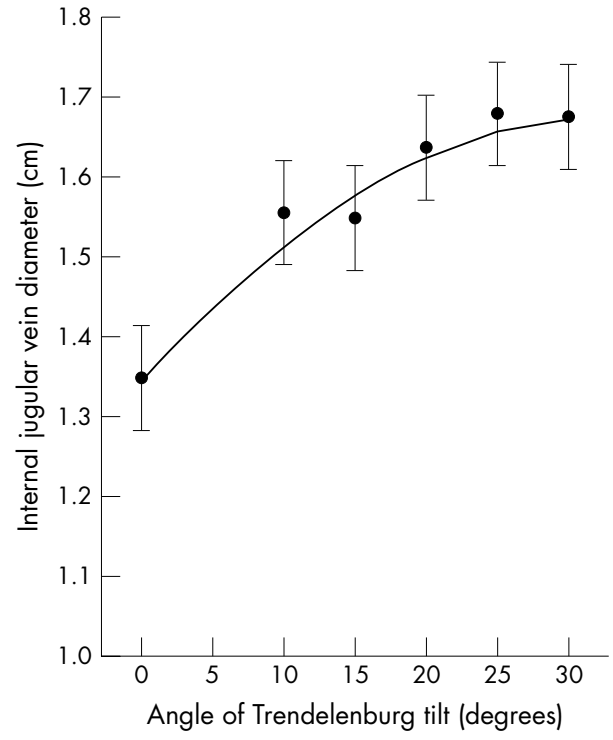

Figure 1 Relationship of the internal jugular vein diameter to various angles of Trendelenburg tilt. Linear and quadratic polynomial terms in tilt angle were found to be significant permitting the fitting of a smooth curve to describe the relationship between diameter and tilt angle.

reduce the rates of complication and improve the rate of cannulation success. In the absence of ultrasound guidance most clinicians would use Trendelenburg positioning, though this can be poorly tolerated and associated with increased complications (such as risk of raised intracranial pressure) in some patient populations..$^{5-7}$

Our study demonstrates that $10^{\circ}$ Trendelenburg significantly increases IJV diameter in healthy adults, although this did vary greatly between individuals, with an actual distension of between $1.2 \mathrm{~mm}$ and $7.0 \mathrm{~mm}$. There was a nonsignificant trend to further increases with greater angles of tilt, maximal at $25^{\circ}$; however, this increase was relatively small, with a mean of $1.2 \mathrm{~mm}$. Larger studies may demonstrate significant increases at angles of tilt greater than $10^{\circ}$, although our subjects reported discomfort with angles greater than $10^{\circ}$. Furthermore, most ED trolleys tilt only to $10-15^{\circ}$. Hence, greater angles of tilt are not only impractical in unstable patients but may also be of little benefit.

Armstrong et $a l^{3}$ Verghese et $a l^{8}$ and others ${ }^{9-12}$ have demonstrated that various manoeuvres are effective in increasing IJV diameter in healthy adults. The Valsalva technique is particularly effective and a combination of techniques has been advocated. However, the Valsalva technique is often impractical in critically ill patients.

In the present study, the effect of respiratory cycle on IJV diameter was overcome by measuring maximal diameter over three respiratory cycles for each subject at each angle of tilt. Interobserver variability was eliminated because only one researcher carried out the measurements.

\section{CONCLUSION}

Increasing the degree of Trendelenburg tilt increases the lateral diameter of the IJV. Even a $10^{\circ}$ tilt is effective. The cumulative effect of tilt (that is, the stretching effect of the previous angle) is not significant. Ultrasound guided cannulation is ideal but in its absence Trendelenburg tilt will increase IJV diameter and may improve the chance of successful cannulation. While $25^{\circ}$ may achieve optimum distension, this is impractical and may be detrimental. There
Table 1 Internal jugular vein (IJV) diameter at various angles of Trendelenburg tilt

\begin{tabular}{lllr}
\hline $\begin{array}{l}\text { Trendelenburg } \\
\text { angle }\end{array}$ & $\begin{array}{l}\text { Mean IJV diameter } \\
(\mathbf{m m})\end{array}$ & $\mathbf{9 5 \%} \mathbf{C l}$ & \multicolumn{1}{c}{ Range } \\
\hline 0 & 13.5 & 12.8 to 14.1 & $9.9-23.5$ \\
10 & 15.5 & 14.9 to 16.1 & $11.2-25.7$ \\
15 & 15.5 & 14.8 to 16.1 & $10.7-26.2$ \\
20 & 16.4 & 15.7 to 17.0 & $10.9-28.2$ \\
25 & 16.7 & 16.1 to 17.4 & $11.7-25.4$ \\
30 & 16.7 & 16.1 to 17.4 & $10.9-26.5$ \\
\hline
\end{tabular}

was no benefit in tilting the patient to a steeper angle prior to settling at a lesser angle to carry out the procedure. There did not appear to be any "stretching" of the IJV to allow greater distension at a lesser angle.

Trendelenburg tilt is used in IJV cannulation to optimise conditions for successful cannulation. Our results show that IJV distension shows marked inter-subject variability and that minimal increases in diameter may occur. Although this may be statistically significant, is it clinically significant? It is difficult to say. This study was not designed to assess the success of cannulation, but it highlights a significant problem with blind cannulation in that we do not know, in an individual patient, how the IJV will respond. What we can do is provide optimum conditions to facilitate cannulation.

We recommend that practitioners use $10^{\circ}$ of tilt when attempting central venous cannulation in the absence of real time ultrasound. However this technique assumes normal anatomy and therefore real time ultrasound is preferable.

\section{Authors' affiliations}

S Clenaghan, R E McLaughlin, C Martyn, S McGovern, J Bowra, Ulster Hospital, Belfast, Northern Ireland

Competing interests: none declared

\section{REFERENCES}

1 National Institute for Clinical Excellence. Technology Appraisal Guidance No. 49. Guidance on the use of ultrasound locating devices for placing central venous catheters. London: September, 2002. Available at: www.nice.org.uk/ pdf/ultrasound 49_GUIDANCE.pdf (accessed 20 September 2005).

2 Lee A, Clenaghan S, McLaughlin R, et al. Emergency department ultrasound: ready or not. Poster presentation at British Association for Accident and Emergency Medicine meeting.

3 Armstrong PJ, Sutherland R, Scott DH. The effect of position and different manoeuvres on internal juggler vein diameter size. Acta Anaesthesiol Scand 1994;38:229-31

4 Cochran WG, Cox GM. Experimental Designs. 2nd edn. 133-4.

5 Trautner H, Greim CA, Arzet $\mathrm{H}$, et al. Ultrasound-guided central venous cannulation in neuropaediatric patients to avoid measures causing potential increase in brain pressure. Anaesthetist 2003;52:115-19.

6 Foltz EL, Blanks JP. Symptomatic low intracranial pressure in shunted hydrocephalus. J Neurosurg 1998;68:401-8.

7 Mavrocordatos P, Bissonnette B, Ravussin P. Effects of neck position and head elevation on intracranial pressure in anaesthetized neurosurgical patients: preliminary results. J Neurosurg Anesthesiol 2000;12:10-14.

8 Verghese ST, Nath A, Zenger D, et al. The effects of the simulated Valsalva maneuver, liver compression, and/or Trendelenburg position on the crosssectional area of the internal jugular vein in infants and young children. Anesth Analg 2002;94:250-4.

9 Schreiber SJ, Lambert UK, Doepp F, et al. Effects of prolonged head-down tilt on internal jugular vein cross-sectional area. $\mathrm{Br} J$ Anaesth 2002;89:769-71

10 Hayashi $\mathbf{H}$, Ootaki C, Tsuzuku M, et al. Respiratory jugular venodilation: its anatomic rationale as a landmark for right internal jugular vein puncture as determined by ultrasonography. J Cardiothorac Vasc Anesth $2000 ; 14: 425-7$.

11 Shimizu T, Miyabe M, Takahashi H, et al. [Change of cross-sectional area of the right internal jugular vein: effect of Trendelenburg position and Valsalva maneuver] [Article in Japanese]. Masui 2001;50:1186-8.

12 Bazaral M. Harlan S. Ultrasonographic anatomy of the internal jugular vein relevant to percutaneous cannulation. Crit Care Med 1981;9:307-10. 